\title{
Non-otogenic cerebral abscess ${ }^{1}$
}

\author{
GRAHAM MARTIN
}

From the Alfred Hospital, Melbourne, Australia

SUMMARY Non-otogenic cerebral abscess is becoming relatively more frequent compared with otogenic. It arises in a setting of sepsis, trauma, or congenital heart disease. The onset presents a meningo-encephalitic picture, often with two stages, followed by epilepsy and/or a hemiparesis. The best investigations are an EEG and, probably, an isotope scan. The angiogram may be misleading as $40 \%$ may not have a midline shift. 'Acute localized non-suppurative encephalitis' seems to be more frequent than in the past. Antibiotics and aspiration appear to be the best treatment.

The large number of abscesses due to chronic otitis media in reported series tends to obscure the distinctive clinical features of abscesses arising from other causes. Yet in a recent British series (Garfield, 1969) they were responsible for $45-50 \%$ of the mortality.

In Australia chronic otitis media is uncommon and otogenic cerebral abscess has become even less common with the widespread use of antibiotics. This series of cerebral abscesses observed at one teaching hospital in Melbourne during the last 15 years does not contain a single otogenic abscess.

\section{MATERIAL}

This consists of 28 abscesses treated at the Alfred Hospital, Melbourne, in the last 15 years. This is a general teaching hospital, and, except that it does not have a large paediatric department, the composition of this series should be representative of cerebral abscess in an Australian community. It is possible that congenital heart disease is somewhat overrepresented because the hospital has a large cardiothoracic outpatient clinic. The abscesses were treated by five different surgeons working in the one unit over the years. Subdural abscesses are not included as they present some different features (Jennett, 1969).

AETIOLOGY Congenital heart disease (eight cases) was the most common single cause $(29 \%)$, followed by trauma, seven cases $(25 \%)$. Metastatic abscesses from the lung and elsewhere accounted for four

1 Work supported by the Edward Wilson Memorial Research Scholarship. cases, sinusitis for two, and the cause was unknown in six cases. Operative infections accounted for two. There were no abscesses due to middle ear disease. Congenital heart disease was associated with cyanosis due to a right to left shunt, except once when the lesion was mitral incompetence with pulmonary hypertension.

The organism was a non-haemolytic streptococcus in nine cases $(32 \%)$ and of these five were anaerobic. A staphylococcus was grown in five $(18 \%)$, anaerobic streptococcus and bacteroides in three $(11 \%)$. Actinomyces and pneumococcus were each seen once. The organism was unknown in nine cases $(32 \%)$. Thirteen sensitivities were available and penicillin with streptomycin would cover the organism in every case. Two others were successfully treated with penicillin and sulphonamides and one with penicillin and tetracycline, though culture was sterile.

SITES OF ABSCESSES Eleven $(39 \%)$ were frontal, eight $(24 \%)$ temporal, five $(18 \%)$ parietal, two $(7 \%)$ multiple, and one $(3 \%)$ cerebellar. Despite the overall preponderance of frontal abscesses, of those associated with congenital heart disease six $(75 \%)$ were in the temporoparietal region. Abscesses arising from trauma were usually related to the site of trauma. There was no predominance of one side over the other.

CLINICAL CONDITION Though the background was present for a cerebral abscess in some-for example, the presence of an abscess elsewhere or cyanotic congenital heart disease-the background did not apparently affect the mode of onset of the abscess.

The most common onset was a meningo-encephalitic picture of headache and drowsiness, sometimes 
with neck stiffness and fever. This was seen in 17 of the patients $(61 \%)$. It was combined with the development of a hemiparesis in $12(43 \%)$ and epilepsy in eight $(29 \%)$, usually generalized epilepsy but in two a focal epilepsy.

A two-stage history, of minor malaise, sometimes with a remission, followed by a major meningoencephalitic syndrome occurred in eight patients $(29 \%)$. Three, in fact, improved so much that they were discharged from hospital before the second stage occurred.

Fever above $38^{\circ} \mathrm{C}\left(100.4^{\circ} \mathrm{F}\right)$ on presentation was a feature of nine patients $(32 \%)$.

The blood white cell count was available in 19 patients. In 13 of these $19(69 \%)$ it was above 9,000 and in five $(26 \%)$ above 13,000 per c. $\mathrm{mm}$. The erythrocyte sedimentation rate (ESR) was done six times and was usually elevated as was the neutrophil alkaline phosphatase in five patients.

INVESTIGATIONS Lumbar puncture There was nothing characteristic about the results. The pressures ranged from normal to $400 \mathrm{~mm}$ of water, the cell counts in the cerebrospinal fluid (CSF) from normal to 1,000 white cells $/ \mathrm{mm}^{3}$ and the protein from normal to $390 \mathrm{mg} / 100 \mathrm{ml}$.

One patient developed a pressure cone after lumbar puncture.

Electroencephalography (EEG) This was of localizing value in $11(79 \%)$ out of the 14 in whom it was done. In one patient it was initially normal but subsequently became abnormal.

Of those in whom it was not of localizing value, one had bilateral abscesses and another a cerebellar abscess.

Angiography This was done in 19 patients. In five patients it was normal several weeks after the onset $(26 \%)$. In the other 14 it was of localizing value, though three of these did not show a midline shift. Thus the absence of a midline shift was noted in eight patients $(39 \%)$ out of the 26 with unilateral supratentorial abscess. Five of these eight patients had congenital heart disease. Of those without a midline shift, three were known to have developed a shift later, after one to three weeks. One further patient had a frontal lobe abscess with a midline pineal gland but some shift of the anterior cerebral artery, and another had an undiagnosed cerebellar abscess.

TREATMENT Eleven patients had repeated aspiration and six $(55 \%)$ died. Five patients had a drain tube inserted and two died. Three had a craniotomy and drainage and all died. One patient had a cranio- tomy and excision and survived. Two patients were $\bar{z}$ treated with antibiotics alone; one survived, the $\stackrel{\mathbb{D}}{\subseteq}$ other coned after a lumbar puncture.

The abscess was undiagnosed in three patients all of whom died. In another four patients, who all died, the lesion was biopsied by craniotomy for sus- ve pected cerebral tumour.

MORTALITY Congenital heart disease and traumatic. abscesses had the best prognosis. Three out of eight $\vec{F}$ patients $(37 \%)$ died with congenital heart disease $\stackrel{\mathcal{O}}{\stackrel{5}{+}}$ and three out of seven $(43 \%)$ with traumatic abscesses.

The remaining miscellaneous group had a very high mortality, 11 out of 14 patients died $(78 \%)$.

\section{DISCUSSION}

In this series the overall mortality is $61 \%$. Further reduction in the mortality of cerebral abscess will depend partly on the successful management of non-otogenic cerebral abscess DIAGNOSIS The setting in which non-otogenif응 cerebral abscesses occur is that of sepsis, trauna (often with sepsis), or cyanotic congenital heart disease. The sepsis is not exclusively in the lung; it may be in the cranial vault, sinuses, or else where.

Though some abscesses were associated with. congenital heart disease, none occurred during the course of subacute bacterial endocarditis. It has been noted before that cerebral abscess does not seem to complicate the course of subacute $\frac{\mathbb{Q}}{\circ}$ bacterial endocarditis (Newton, 1956).

The trauma was usually severe, such as a depressed fracture, but sometimes it was minor, no more than a blow to the head while playing? football.

The onset is usually a meningo-encephalitic picture of drowsiness, headache, and neck stiff- 0 ness. This may be associated with a hemiparesis and/or a generalized or focal epileptic fit.

A third of the cases have a two-stage history of preceding malaise with complete or partial remission for a few days.

Fever may be quite high, despite the commonly repeated statement that cerebral abscesses have a low fever. In $32 \%$ of cases it was over $38^{\circ} \mathrm{C}$ $\left(100 \cdot 4^{\circ} \mathrm{F}\right)$. 
Papilloedema was absent in the majority of patients.

INVESTIGATIONS Traditionally a plain skull and chest radiograph are the first investigations. While these may show a pulmonary abscess, a pineal shift or a fracture running into a nasal sinus, these radiographs may be perfectly normal and yet the patient still have a cerebral abscess.

An abscess may be present without a midline shift; consequently neither a midline pineal shadow on radiography nor a normal echoencephalogram will exclude an abscess. This point will be dealt with further when angiography is discussed.

The EEG was the most valuable investigation for localizing the abscess. In $79 \%$ it was localized by high voltage waves, 1-3 per second, with phase reversal. Only one out of the 14 EEGs was completely normal. Thus it may be said that a normal EEG makes an abscess unlikely.

Angiography was helpful in $75 \%$ of patients in whom it was done. But it is important to notice the $25 \%$ who had an abscess with a normal angiogram. A further three patients $(15 \%)$ did not have a midline shift, though the angiogram localized the abscess. Of the five patients who did not have a midline shift three were subsequently known to have developed a shift one to three weeks later. Schurr (1951) first described the absence of a midline shift in patients with abscesses. Presumably these were early abscesses in the stage described by Botterell and Drake (1952) and Levy (1963) of 'acute localized non-suppurative encephalitis'. Probably isotope scanning will show these areas of acute non-suppurative encephalitis but we have not enough experience of it yet.

The high incidence of this type of abscess in this series may be because most of the patients were admitted primarily to this hospital and were not simply the survivors who were fit to be transferred to a neurosurgical unit. This may also account for the high mortality in this series. Levy (1963) commented on the high mortality of abscesses among Rhodesian Europeans, most of whom saw a neurosurgeon, compared with Rhodesian Africans among whom only the survivors were seen. Garfield (1969) pointed out that, despite increased numbers of neuro- surgeons, the mortality of abscesses was not falling, probably because more severely ill patients were being referred.

Other investigations were not of much help. A lumbar puncture precipitated the death of one patient out of the 10 in whom it was done. Our experience confirms that of Locoge and Cumings (1958) that there are no characteristic CSF findings and that of Duffy (1969) that it is a dangerous investigation.

The white cell count in the blood was variable and of no diagnostic value, ranging from normal to 28,000 per c. $\mathrm{mm}$. A monocytosis was noticed sometimes.

TREATMENT Aspiration of the abscess was frequently the final investigation. Aspiration, repeated as needed, or the insertion of a drain tube were the most successful treatments.

The mortality was high when the diagnosis was missed. An excision or open biopsy under the impression that the lesion was a tumour produced a mortality of three out of four cases.

Antibiotics were an important adjunct to treatment; one patient survived with antibiotic treatment alone. Penicillin and streptomycin were the antibiotics of choice. There were 13 sensitivities which were available; penicillin and or streptomycin would have been adequate treatment for all of them. The organism was also sensitive to sulphonamides in $46 \%$ of the instances.

It is suggested that patients be started on penicillin, streptomycin, and sulphonamides immediately pus has been obtained for culture.

CONCLUSION As otogenic abscess becomes rarer, the recognition of non-otogenic abscess becomes more important and, as neurosurgeons become more numerous, patients with abscesses are referred earlier, and are often more sick.

A non-otogenic abscess may be suspected when a meningo-encephalitic illness with hemiparesis or epilepsy occurs in a setting of sepsis elsewhere, trauma, or congenital heart disease. It is confirmed by the EEG, an angiogram (allowing for its limitations), and aspiration of the pus. The latter is the final investigation and also the best treatment. 


\section{REFERENCES}

Botterell, E. H., and Drake, C. G. (1952). Localized encephalitis, brain abscess and subdural empyema, 1945-1950. Journal of Neurosurgery, 9, 348-366.

Duffy, G. P. (1969). Lumbar puncture in the presence of raised intracranial pressure. British Medical Journal, 1, 407-409.

Garfield, J. (1969) Management of supratentorial intracranial abscess: a review of 200 cases. British Medical Journal, 2 7-11.

Jennett, W. B. (1969). Supratentorial intracranial abscess. (Letter.) British Medical Journal, 2, 310-311.
Levy, L. F. (1963). Intracranial abscess: the shape of things to come? British Medical Journal, 1, 1455-1458.

Locoge, M., and Cumings, J. N. (1958). Cerebrospinal fluid in various diseases. British Medical Journal, 1, 618-620.

Newton, E. J. (1956). Haematogenous brain abscess in cyanotic congenital heart disease. Quarterly Journal of Medicine, 25, 201-220.

Raimondi, A. J., Matsumoto, S., and Miller, R. A. (1965). Brain abscess in children with congenital heart disease. Journal of Neurosurgery, 23, 588-595.

Schurr, P. H. (1951). Cerebral angiography in abscess of the temporal lobe. British Journal of Surgery, 39, 156-165. 